\title{
Erratum to: Whole Genome DNA Methylation Analysis of Brachypodium distachyon Using Next-Generation Sequencing (BS-seq)
}

I-Hsuan Lin

Erratum to:

Chapter 17 in: Gaurav Sablok et al. (eds.),

Brachypodium Genomics: Methods and Protocols,

Methods in Molecular Biology, vol. 1667,

Chapter 17 was originally published without the author's corrections but the current 11 version has been updated with those corrections included.

The online version of the updated original book can be found at https://doi.org/10.1007/978-1-4939-7278-4_17 